\title{
Oral Health Status of South-Eastern Iranian Children with Acute Lymphoblastic Leukemia
}

\author{
Marzieh Karimi Afshar ${ }^{1}$, Zahra Farahmandinia², Molook Torabi³, Fatemeh Parivar ${ }^{4}$ \\ ${ }^{1}$ Department of Orthodontic, Dental School, Kerman University of Medical Sciences, Kerman, Iran. ${ }^{2}$ Department of \\ Paediatric, Medical School, Kerman University of Medical Sciences, Kerman, Iran. ${ }^{3}$ Department of Oral Pathology, \\ Kerman Social Determinants on Oral Health Research Center and School of Dentistry, Kerman University of Medical \\ Sciences, Kerman, Iran. ${ }^{4}$ Department of Dental, Dental School, Kerman University of Medical Sciences, Kerman, Iran.
}

\section{ABSTRACT}

\section{BACKGROUND}

Acute leukemia is characterized by the progressive excessive production of leukocytes in the bone marrow as well as the arrival of immature cells into the peripheral blood. The present study aimed to evaluate the oral hygiene status in patients with acute leukemia.

\section{METHODS}

The present study included 50 ALL patients referred to the affiliated oncology centers of Kerman University of Medical Sciences from April to December 2015 and 52 healthy children. Data collected from the oral examination included the $\mathrm{DMFT} / \mathrm{dmft}$ and plaque indexes and the presence of mucositis. Information about the diseases, blood test results, the patients' age, and the type of drug was obtained from medical records. Data was analysed through SPSS software, T-test and MannWhitney test at a significance level of 0.05 .

\section{RESULTS}

Grade 1 and 2 mucositis was observed in $6(0.12 \%)$ and $1(0.2 \%)$ patients respectively. The plaque index was significantly higher in patients than the healthy group ( $\mathrm{p}=0.033$ ). The average missing teeth was higher in the ALL group, but it was not statistically significant. There were no statistically significant differences between the individuals with mucositis and those without mucositis $(p=0.503)$. There were no statistically significant differences between the patients and healthy group in terms of the mean DMFT/dmft index.

\section{CONCLUSIONS}

There were no statistically significant differences between the patients and healthy group in DMFT/dmft index. Statistically significant differences were seen between patients and healthy groups in terms of OHI-S index.

\section{KEY WORDS}

Leukemia, Oral Health, Mucositis, DMFT/dmft
Corresponding Author:

Molook Torabi,

Department of Oral Pathology,

Dental School, Shafa Street,

Kerman, Iran.

E-mail: m.torabi.p@gmail.com

DOI: $10.14260 /$ jemds/2020/19

Financial or Other Competing Interests: None.

How to Cite This Article:

Afshar MK, Farahmandinia Z, Torabi M, et al. Oral health status of South-Eastern Iranian children with acute lymphoblastic leukemia. J. Evolution Med. Dent. Sci. 2020;9(02):86-90, DOI:
Submission 25-09-2019,

Peer Review 25-12-2019,

Acceptance 03-01-2020,

Published 13-01-2020. 


\section{BACKGROUND}

Leukemia is a disease which is characterized by the progressive and excessive production of leukocytes in the bone marrow and arrival of immature cells in the peripheral blood. ${ }^{1}$ The aetiology of this disease is not well understood. Most studies have investigated the factors such as the viruses, retroviruses, genetic factors, radiation and chemical agents. 2,3,4,5,6 Leukemia includes different sections based on the type of involved cells, duration of disease, and its clinical characteristics. Acute lymphoblastic leukemia (ALL) accounts for about $80 \%$ of leukemia which mostly occurs in the children. The most common oral symptoms of leukemia include the gingival bleeding and hyperplasia, opportunistic infections, and bone changes. Since the chemotherapy also affects normal cells, the symptoms can be seen during the treatment and may be also intensified. Dentists can diagnose its initial symptoms especially in the acute phase of the disease. ${ }^{1}$ Mucositis is the most important oral problem of patients in the chemotherapy phase.1,7 Mucositis is characterized by the redness and oral ulcers, and begins 4-7 days after the chemotherapy. ${ }^{8}$ In patients with leukemia, the inflammatory periodontal disease and oral mucositis have significantly higher than the healthy group. ${ }^{9}$ Pels et al. (2012) found that the ALL children had significantly better oral hygiene than the healthy group, but their gingival index (GI) was more than the healthy group. ${ }^{2}$ It is found that the ALL children are at the higher risk of tooth decay than the healthy group; and the root malformations, dental agenesis, microdontia and enamel dysplasia are more common among the children with this disease. ${ }^{10,11} \mathrm{~A}$ study reported the higher rates of dental caries in the ALL children than the normal population. ${ }^{12}$ It is shown the oral hygiene status in the ALL children in different phases of treatment and found that the DMFT/DMFT indices were much further during the maintenance phase than other phases in. ${ }^{13} \mathrm{~A}$ study reported the prevalence of oral lesions in the ALL children according to the following order: Gingivitis $91.84 \%$, tooth decay $81.63 \%$, mucositis $38.77 \%$, periodontitis $16.32 \%$ and $18.36 \%$ angular chilitis. They concluded that the ALL and poor oral hygiene increased the patients' susceptibility to candidiasis and gingivitis. ${ }^{14}$ Mucositis was the most common problem in patients with the ALL; and maxillary posterior buccal mucosa was the most common site. Results of conducted studies by the above-mentioned researchers indicated that the oral manifestations were more common in the ALL patients than the healthy group and were also more prevalent in the induction phase of treatment than other phases. ${ }^{3}$ Considering dental and oral problems in patients with leukemia, dentists should be aware of the lesions which are caused by this disease and prescribe medications for its treatment to improve their oral health. The present study was conducted to determine the children's dental and oral problems.

\section{METHODS}

The present study was a cross-sectional study which was conducted on the ALL patients who referred to the affiliated Oncology Centers of Kerman University of Medical Sciences during the April-December 2015 and desire to participate in research and 50 healthy children. After obtaining the necessary permissions, a senior trained dental student, with necessary skills, referred to the related centers for examination. Before the examination, the purpose of study was explained to patients/parents and their verbal consent was obtained. Finally, 50 patients examined. ${ }^{15}$

The oral examination (Decay, Missing, and Filling teeth) included DMFT/DMFT indices and the plaque index. DMFT/DMFT indices were evaluated according to the World Health Organization (WHO) criteria. ${ }^{16}$

\section{Oral Hygiene Status by Plaque Index (Silness and Loe)}

- Score $0=$ No plaque in the gingival area.

- Score 1= A film of plaque adheres to the free gingival margin and adjacent area of the tooth and it is only diagnosed while probing all accessible tooth surfaces.

- $\quad$ Score 2= Moderate accumulation of soft deposit within the gingival pocket, or the tooth and gingival margin than is visible to the naked eye.

- Score 3= Abundance of the soft matter within the gingival pocket or on the tooth and gingival margin. ${ }^{17}$

\section{Mucositis Assessment Based on WHO Criteria}

- $\quad$ Score $0=$ No mucositis symptoms.

- $\quad$ Score 1= Redness, Erythema and burning sensation.

- Score 2= Erythema and ulcers. Patient can swallow solid food.

- Score 3= Ulcers and redness. Patients are unable to swallow solid food.

- $\quad$ Score $4=$ Ulcers and pseudomembranous that prevent eating. ${ }^{18}$ Information on diseases such as step therapy, blood tests and patients' age were extracted from patients' medical records. Finally, the personal hygiene practices were taught to the participants. In the present group, the healthy group covered the individuals at the same age range of patients without leukemia disease or other systemic disease affecting these indices. The above-mentioned indices were then measured.

\section{Statistical Analysis}

Data entered into the checklists and later into the computers to be analysed through the $\mathrm{T}$ and Mann-Whitney tests in the SPSS software (ver. 21) at the significance level of $0.5 \%$. Normalized data distribution performed.

\section{Ethical Consideration}

This project was approved by the Ethics Committee of Kerman University of Medical Sciences under the code of IR.KMU.REC.1395.36.

\section{RESULTS}

Results of the present study, which was conducted on 50 patients with acute lymphoblastic leukemia and underwent the treatment process and 52 individuals in the healthy group, are as follows: There were 34 male $(68.00 \%)$ and 16 female patients $(32.00 \%)$. There were 30 females $(57.7 \%)$ and 22 males $(42.3 \%)$ in the healthy group. (Table 1$)$. In the patient group, $13(0.26 \%), 14(0.28 \%)$ and $23(0.46 \%)$ patients were respectively in the induction, consolidation and 
maintenance phases. A total of 15 (30\%), 22 (44\%) and 13 patients (26\%) were treated with cyclophosphamidecytarabine, VCR-mercaptopurine-methotrexate and vincristine- daunorubicin -asparaginase respectively. After examination of under-treatment patients, 43 patients did not have the mucositis, 6 individuals had the grade- 1 mucositis $(0.12 \%)$; and 1 patient $(0.2 \%)$ had the grade- 2 mucositis. The mean and standard deviation of the patients' WBC and RBC and blood platelets are presented in the Table 2 for both genders. The mean and standard deviation of the DMFT/dmft status of patients and healthy groups are presented in the Table 3. The number of filled teeth in the healthy group was significantly higher $(p=0.033)$ according to the filled teeth index. Furthermore, the plaque index was significantly higher in the patients than the healthy group $(\mathrm{p}=0.033)$. The average extracted tooth due to the decay in the treatment group was higher than the healthy group, but it was not statistically significant. There were not any statistically significant differences between individuals with and without mucositis in terms of the mean plaque index $(p=0.503)$.Differences between healthy and patients' groups based in plaque index is shown in the Table 4 .

\begin{tabular}{|c|c|c|c|c|}
\hline \multirow{2}{*}{ Variables } & \multicolumn{2}{|c|}{ Female } & \multicolumn{2}{c|}{ Male } \\
\cline { 2 - 5 } & Number & Percentage & Number & Percentage \\
\hline Patients & 16 & 32.00 & 34 & 68.00 \\
\hline Healthy & 30 & 57.69 & 22 & 42.31 \\
\hline Total & 46 & 45.09 & 56 & 54.91 \\
\hline Table 1. Frequency Distribution of Patients and Healthy Groups in \\
Terms of Gender \\
\hline
\end{tabular}

\begin{tabular}{|c|c|c|c|c|c|c|c|c|}
\hline \multirow[b]{2}{*}{ 竞 } & \multicolumn{2}{|c|}{ RBC } & \multicolumn{2}{|c|}{ WBC } & \multicolumn{4}{|c|}{ Platelets } \\
\hline & $\sum_{\Sigma}^{\overparen{\Xi}}$ & $\dot{\dot{\omega}}$ & $\sum_{\Sigma}^{\overparen{\Xi}}$ & में & $\stackrel{\Xi}{\tilde{\Xi}}$ & $\dot{\vec{s}}$ & : & 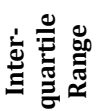 \\
\hline Male & 3.83 & 0.88 & 3.34 & 1.34 & 131.71 & 249.39 & 142.75 & 145.34 \\
\hline Female & 4.05 & 1.47 & 4.25 & 2.09 & 148.64 & 196.76 & 158.00 & 162.21 \\
\hline Total & 3.90 & 1.10 & 3.64 & 3.40 & 138.16 & 232.14 & 248.00 & 251.13 \\
\hline \multicolumn{9}{|c|}{$\begin{array}{c}\text { Table 2. Mean and SD of the WBC and RBC and } \\
\text { Platelets for both Genders }\end{array}$} \\
\hline
\end{tabular}

\begin{tabular}{|c|c|c|c|c|}
\hline Variable & Group & Mean & S.D. & p-Value \\
\hline \multirow{2}{*}{$\mathrm{D} / \mathrm{d}$} & Patients & 2.30 & 2.72 & \multirow{2}{*}{$\mathrm{p}>0.05$} \\
\hline & Healthy & 2.96 & 1.83 & \\
\hline \multirow{2}{*}{$\mathrm{M} / \mathrm{m}$} & Patients & 1.40 & 1.10 & \multirow{2}{*}{$\mathrm{p}>0.05$} \\
\hline & Healthy & 0.53 & 0.91 & \\
\hline \multirow{2}{*}{$F / f$} & Patients & 0.26 & 0.72 & \multirow{2}{*}{0.033} \\
\hline & Healthy & 0.91 & 0.63 & \\
\hline \multirow{3}{*}{ DMFT/dmft } & Patients & 4.01 & 3.60 & \multirow{3}{*}{$\mathrm{p}>0.05$} \\
\hline & Healthy & 4.13 & 2.25 & \\
\hline & Healthy & 0.79 & 0.61 & \\
\hline \multicolumn{5}{|c|}{$\begin{array}{c}\text { Table 3. Correlation between DMFT/dmft Status of } \\
\text { Patients and Healthy Groups }\end{array}$} \\
\hline
\end{tabular}

\begin{tabular}{|c|c|c|c|c|c|}
\hline Variable & Group & Median & IQR & Mean of Rank & p-Value \\
\hline \multirow{2}{*}{ Plaque index } & Patients & 1 & 1 & 57.28 & \multirow{2}{*}{0.011} \\
\cline { 2 - 5 } & Healthy & 1 & 0 & 43.72 & \\
\hline \multicolumn{7}{|c|}{ Table 4. Difference between Patients and Healthy Group } \\
According to Plaque Index
\end{tabular}

\section{DISCUSSION}

Leukemia is the most common malignancy in the children; and the paediatric dentists play important roles in the prevention, treatment and healthy of oral and dental problems which affect the children health and quality of life before, during and after the treatment. ${ }^{8}$ Acute lymphoblastic leukemia (ALL) often occurs in children and accounts for almost $80 \%$ of leukemia. The increased gingival volume and bleeding are the most common oral symptoms. ${ }^{1}$

Results of the present study, which was conducted on 50 patients with the ALL, indicated that $68 \%$ of patients were male, and these results were consistent with results of research by Carrillo et al. who found that there were a larger number of men with hematologic malignancies among haematology-oncology patients in São Paulo, Brazil.19 Mucositis was observed in $14 \%$ of patients in the present study. Results of research by Soares et al. (2011) on 17 children with the ALL in Brazil indicated that 5 patients (29.41\%) had mucositis. ${ }^{20}$ In a research by Toress et al. (2010) in Mexico, the mucositis was observed in $38.77 \%$ of the ALL patients. ${ }^{14}$ Subramaniam et al. (2008) reported that the frequency of mucositis was $20.6 \%$ in the Indian patients with the ALL. ${ }^{3}$ Mendonca et al. (2012) found that the HSV and Candida were associated with the increased mucositis in patients with the ALL. ${ }^{21}$ Obviously, the higher percentage of mucositis was seen in the studied patients in the abovementioned research compared to the present study; and it was probably due to the study design and sample size. Considering the fact that $46 \%$ of patients were in the maintenance phase, it could be a reason for the decreased mucositis rate in the present study. Furthermore, there were not any statistically significant differences between the type of prescribed drug and the incidence of mucositis. Studies found that the average time of development of mucositis lesions and ulcers was 10-16 days after the initiation of chemotherapy; and the oral problems did not depend on the chemotherapy protocol.22,23,24 It was indicated that the mucositis developed in children with leukemia to a large extent due to the reduced levels of S-IgA, myeloperoxidase, salivary peroxidase and $50 \%$ reduction in the amounts of salivary proteins compared with the without-mucositis ALL patients. ${ }^{25}$

Results of conducted study by Soares et al. indicated that the use of $0.12 \%$ Chlorhexidine and the oral rinse solution reduced the oral mucositis and pathogens in children with the ALL. ${ }^{20}$ In the present study, the average GI Index was $0.9 \pm 0.1 \%$ in patients with the ALL. There were not any statistically significant differences between the patients and healthy group. Therefore, the patients had higher OHI-S index; and this was inconsistent with results of conducted research by Pels who argued that the healthy subjects had better indexes. ${ }^{2}$ This inconsistency could be due to the fact that $26 \%$ of patients were in the induction phase in the present study. Mucositis is one of the main problems in the multi-drug chemotherapy treatment and it can be associated with a less-compliance rate with the oral hygiene. There was not any correlation between the plaque rate and haematological criteria. In a study on the Santa Maria patients in Brazil, Angst et al. reported the plaque index of $1.32 \pm 0.54$ and there was not any significant relationship between the platelets with dental plaque; and it was consistent with results of the present study. ${ }^{26}$ In the present study, a total of $85 \%$ of patients suffered from the dental caries. Results of the present study were consistent with results of research by Torres et al. (2012) in Mexico where the decay was observed in $81.63 \%$ of patients with the ALL. ${ }^{14}$ In the present study, there were not any statistical significant differences between 
patients with the ALL and the healthy group in terms of the DMFT/DMFT index, but the standard deviation of index was higher in patients than the healthy group. Cubukçu observed that there were not any statistical differences between the patients, who had long survived cancer, and the healthy group in terms of the mean DMFT Index. ${ }^{27}$ In a 10-year review on the individuals, who survived the ALL and AML, Lauritano et al. found that the DMFT index was higher in the treated patients than the healthy group. ${ }^{10}$ In the present study, there were statistical significant differences between the patients and healthy group in terms of the $\mathrm{F}$ index (restored tooth). The healthy group had a higher number of restored teeth; and this was inconsistent with findings of research by Nemeth ${ }^{11}$ who reported that the healthy group had more restored teeth; and it was probably due to the fact that Nemeth's study was conducted on the children who were previously treated. In the present study, the lower number of filled teeth might be attributed to the fact that the patients' parents were more involved in the disease and its treatment, and thus they paid great attention to their oral and dental health. In addition to the antineoplastic therapy, the special attention should be paid to the children's oral dental needs with blood diseases. Oral hygiene and dental health status should be periodically monitored through the close collaboration between the oncologists, pediatricians, pediatric dentist and dental hygienist. Despite the fact that the side effects of antineoplastic drugs are not avoidable, the patients' quality of life can be enhanced by compliance with oral and dental hygiene care and prevention of oral diseases.

\section{CONCLUSIONS}

Findings of the present study indicate that most of the ALL patients were male. There were no statistically significant differences between the patients and healthy groups in terms of the DMFT index. There were statistically significant differences between the patients and healthy groups in terms of the OHI-S index.

\section{Limitations}

As dmft/DMFT and plaque index are the most common indices in oral health field, we evaluated these indices. Other indicies were not measured.

\section{ACKNOWLEDGEMENT}

The authors thank the Deputy of Research and Technology at Kerman University of Medical Sciences for approval the present study. The authors thank patients who participated in the study, and the personnel of Afzalipour hospital for their cooperation during the study.

\section{REFERENCES}

[1] Morais EF, Lira JA, Macedo RA, et al. Oral manifestations resulting from chemotherapy in children with acute lymphoblastic leukemia. Brazilian Journal of Otorhinolaryngology 2014;80 (1):78-85.
[2] Pels E, Mielnik-Blaszczak M. Oral hygiene in children suffering from acute lymphoblastic leukemia living in rural and urban regions. Annals of Agricultural and Environmental Medicine 2012;19 (3):529-33.

[3] Subramaniam P, Babu KG, Nagarathna J. Oral manifestations in acute lymphoblastic leukemic children under chemotherapy. Journal of Clinical Pediatric Dentistry 2008;32 (4):319-24.

[4] Hamerschlak N. Leukemia: genetics and prognostic factors. Jornal de Pediatria (Rio J) 2008;84 (Suppl 4):S52-S7.

[5] McNally RJ, Eden TO. An infectious aetiology for childhood acute leukaemia: a review of the evidence. British Journal of Haematology 2004;127 (3):243-63.

[6] Gao F, Chia KS, Machin D. On the evidence for seasonal variation in the onset of acute lymphoblastic leukemia (ALL). Leukemia Research 2007;31 (10):1327-38.

[7] Peterson DE. New strategies for management of oral mucositis in cancer patients. The Journal of Supportive Oncology 2006;4 (2 Suppl 1):9-13.

[8] Xavier AM, Hegde AM. Preventive protocols and oral management in childhood leukemia-the pediatric specialist's role. Asian Pac J Cancer Prev 2010;11 (1):3943.

[9] Javed F, Utreja A, Correa FOB, et al. Oral health status in children with acute lymphoblastic leukemia. Critical Reviews in Oncology/Hematology 2012;83 (3):303-9.

[10] Lauritano D, Petruzzi M. Decayed, missing and filled teeth index and dental anomalies in long-term survivors leukaemic children: a prospective healthyled study. Medicina Oral, Patologia Oral Y Cirugia Bucal 2012;17 (6):e977-80.

[11] Nemeth O, Hermann P, Kivovics P, et al. Long-term effects of chemotherapy on dental status of children cancer survivors. Pediatric Hematology and Oncology 2013;30 (3):208-15.

[12] Hegde AM, Joshi S, Rai K, et al. Evaluation of oral hygiene status, salivary characteristics and dental caries experience in acute lymphoblastic leukemic (ALL) children. Journal of Clinical Pediatric Dentistry 2011;35 (3):319-23.

[13] Azher U, Shiggaon N. Oral health status of children with acute lymphoblastic leukemia undergoing chemotherapy. Indian Journal of Dental Research 2013;24 (4):523.

[14] Ponce-Torres E, Ruíz-Rodríguez MS, Alejo-González F, et al. Oral manifestations in pediatric patients receiving chemotherapy for acute lymphoblastic leukemia. Journal of Clinical Pediatric Dentistry 2010;34 (3):275-9.

[15] Gandhi K, Datta G, Ahuja S, et al. Prevalence of oral complications occurring in a population of pediatric cancer patients receiving chemotherapy. Int J Clin Pediatr Dent 2017;10 (2):166-71.

[16] Organization WH. World Health Organization: oral health surveys, basic methods. Geneva: World Health Organization, 1997.

[17] Ramfjord SP. The Periodontal Disease Index (PDI). Journal of Periodontology 1967;38 (6 Part 2):602-10. 
[18] Pels E. Oral mucositis in children suffering from acute lymphoblastic leukaemia. Contemporary Oncology (Pozn) 2012;16 (1):12-15.

[19] Carrillo C, Vizeu H, Soares-Júnior LA, et al. Dental approach in the pediatric oncology patient: characteristics of the population treated at the dentistry unit in a pediatric oncology Brazilian teaching hospital. Clinics (Sao Paulo) 2010;65 (6):569-73.

[20] Soares AF, Aquino ARLd, Carvalho CH, et al. Frequency of oral mucositis and microbiological analysis in children with acute lymphoblastic leukemia treated with $0.12 \%$ chlorhexidine gluconate. Brazilian Dental Journal 2011;22 (4):312-6.

[21] de Mendonça RM, de Araújo $M$, Levy $\mathrm{CE}$, et al. Prospective evaluation of HSV, Candida spp., and oral bacteria on the severity of oral mucositis in pediatric acute lymphoblastic leukemia. Supportive Care in Cancer 2012;20 (5):1101-7.

[22] Costa EM, Fernandes MZ, Quinder LB, et al. Evaluation of an oral preventive protocol in children with acute lymphoblastic leukemia. Pesquisa Odontologica Brasileira 2003;17 (2):147-50.
[23] De Oliveira Lula EC, de Oliveira Lula CE, Alves CMC, et al. Chemotherapy-induced oral complications in leukemic patients. International Journal of Pediatric Otorhinolaryngology 2007;71 (11):1681-5.

[24] Pinto LP, De Souza LB, Gordón-Núñez MA, et al. Prevention of oral lesions in children with acute lymphoblastic leukemia. International Journal of Pediatric Otorhinolaryngology 2006;70 (11):1847-51.

[25] Karolewska E, Konopka T, Pupek M, et al. Antibacterial potential of saliva in children with leukemia. Oral Surgery, Oral Medicine, Oral Pathology, Oral Radiology and Endodontology 2008;105 (6):739-44.

[26] Angst PD, Dutra DA, Moreira CH, et al. Gingival inflammation and platelet count in patients with leukemia: preliminary results. Brazilian Oral Research 2011;25 (6):544-9.

[27] Çubukçu ÇE, Sevinir B. Dental health indices of long-term childhood cancer survivors who had oral supervision during treatment: a case-healthy study. Pediatric Hematology and Oncology 2008;25 (7):638-46. 